vielleicht erhoffte "Ruhe « in den Gewerkschaften wiederherstellen wird. Einerseits dürfte die Radikalisierung der IGM-Spitze gegenüber dem BAG für die Arbeiter, denen derzeit steigende Lebenshaltungskosten und Arbeitshetze zentrale Probleme sind, nur von zweitrangigem Interesse sein, so daß sie durch wissenschaftliche Tagungen nicht von spontanen Streiks abzubringen sein werden. Andererseits wird die Position linker Gewerkschaftler, die schon immer die repressive Funktion der Rechtsprechung aufgezeigt haben, dadurch, daß der Vorstand sich ihre Kritik nun zu eigen macht, gestärkt - ein für die Veränderung innergewerkschaftlicher Willensbildungs- und Entscheidungsprozesse wichtiges Faktum. Von besonderer Bedeutung war in diesem Zusammenhang die Kritik Loderers in seinem Eröffnungsreferat an dem richterlichen Verbot der spontanen Streiks.

Wenn auch vieles darauf hindeutet, daß dem Kongreß keine grundlegend neue Gewerkschaftsstrategie gegenüber dem Justizapparat folgen wird, so sollte doch gegenüber solchen linken Gewerkschaftstheoretikern, welche die Unwandelbarkeit der Gewerkschaften oder gar ihren sozialfaschistischen Charakter beschwören, folgendes festgehalten werden: mit der Zunahme von Klassenkämpfen, die ohne die Gewerkschaften geführt werden, sind diese gezwungen, zumindest verbal ihre weitgehend staatlichen Imperativen folgende Politik zu revidieren; selbst wenn die Revision nur verbal erfolgt, eröffnet sie denen, die sich um eine Neubestimmung gewerkschaftlicher Strategien bemühen, bessere Handlungsmöglichkeiten. Die Münchner Tagung erweist sich dannit, trotz vieler Mängel, als ein Fortschritt auf dem dornigen Weg zur Veränderung der Gewerkschaften und ihres Verhältnisses zum bürgerlichen Staat.

Rainer Erd

\title{
Richterbund und Vulgär-Marxismus
}

I.

Entschließung des Gesamtvorstands des Landesverbands Hessen des Deutschen Richterbunds zum Wiesbadener Modell der Reformkommission des bessischen Justizministeriums für eine einstufige Juristenausbildung:

Der Gesamtvorstand des hessischen Richterbundes begrüßt angesichts der Unzulänglichkeiten der traditionellen Juristenausbildung, daß eine Reformkommission des hessischen Justizministeriums ein Modell (sog. Wiesbadener Modell) für eine einstufige, Theorie und Praxis verbindende Juristenausbildung erarbeitet hat.

Der Gesamtvorstand wendet sich jedoch mit Nachdruck dagegen, daß auch hier wieder - nur noch unverblümter als in den umstrittenen Rahmenrichtlinien des hessischen Kultusministeriums - ein an sich berechtigtes und dringendes Reform-

\footnotetext{
wenn w wir das Recht in seinen politischen und sozialen Bezügen aufdecken und bewußt machen. Erst vor wenigen Wochen haben war dazu mit unserer wissenschaftlichen Tagung, Streik und Aussperrung einen wesentlichen Beitrag geleistet. Die scheınbar neutrale Positıon des Rechts und der Rechtsprechung wurde dort entlarvt und der Klassencharakter einer Rechtsprechung deutlich., die mit emer konstruierten Sozialadäquanz von Streik und Aussperrung gleichzeıtig eın gleichwertıges Machtverhältnis zwischen Kapıtal und Arbeit unterstellt.*
} 
vorhaben mit einem ideologischen Uberbau befrachtet worden ist, der die gebotene weltanschauliche Neutralität der staatlichen Juristenausbildung mißachtet und damit das Anliegen jeder echten Reform verfälscht.

Anstelle einer kurzen, sachlich - ausgewogenen Beschreibung der zutage getretenen Mängel der überkommenen Juristenausbildung, die eine Reform erforderlich machen, werden in dem Modell sog. "Grundthesen « über die Bewußtseinslage der Juristen in Vergangenheit und Gegenwart aufgestellt, die in ihrer Einseitigkeit und Ausschließlichkeit alle Wesensmerkmale der wieder in Mode gekommenen vulgär - marxistischen Dogmatik aufweisen.

So heißt es über die historische Funktion der Juristen:

»Im konstitutionellen Staat erfüllten die Juristen objektiv die Funktion eines Herrschaftsinstruments. Indem sie die herrschenden Rechtsnormen durchsetzten, dienten sie der Aufrechterhaltung der Klassenherrschaft, der Unterdrückung der Arbeiterklasse."

Die Verfasser dieser Thesen erwecken damit den Anschein, als sei ihre einzige Quelle zur Rechts- und Sozialgeschichte des vergangenen Jahrhunderts das »Kommunistische Manifest « von Karl Marx. Angesichts der Vielfalt der geistigen, materiellen und emotionalen Einflüsse auf Bewußtsein und Handeln der Menschen gerade im konstitutionellen Zeitalter würden derartige apodiktische "Grundthesen " von keinem seriösen nichtmarxistischen Historiker vertreten werden. Es kann nur überraschen, daß derartige undifferenzierte Phrasen aus der vulgär-marxistischen Begriffswelt in ein amtliches Dokument des hessischen Justizministeriums Eingang gefunden haben.

Zum Selbstverständnis des Juristen in der Gegenwart heißt es im Wiesbadener Modell:

"Läßt sich auch schwerlich ein einheitliches, festumrissenes Weltbild des Juristen feststellen, so haben empirische Untersuchungen doch erbracht, daß ein hoher Anteil der Juristen schon vom Elternhaus her in eine Haltung geprägt wird, die an tradierten mittelständischen Wertvorstellungen unreflektiert festhält."

Abgesehen davon, daß auch diese "Grundthese « wiederum ein stark verzerrtes Bild der Wirklichkeit wiedergibt, insbesondere wenn sie gerade auf den Mangel an Reflektion abstellt, wird auch an dieser Stelle wieder deutlich, daß der Jurist einseitig vorwiegend als Mitglied einer Klasse oder einer speziellen Schicht gesehen wird. Die freiheitliche Demokratie dagegen - wie sie im Grundgesetz postuliert ist - lehnt die Auffassung ab, daß Verhalten und Denken des Menschen durch seine Klassenlage eindeutig determiniert ist. -

Der Gesamtvorstand des hessischen Richterbundes fordert daher den hessischen Justizminister auf, zur Wahrung der gebotenen weltanschaulichen Neutralität der staatlichen Juristenausbildung, wie die pluralistische Gesellschaft es erfordert, und des Grundrechts der Freiheit der Lehre die aus dem wirklichkeitsfremden Blickwinkel einer unreflektierten marxistischen Glaubenslehre entnommenen "Grundthesen « aus dem Wiesbadener Modell zu streichen und durch eine ideologiefreie Beschreibung der in der bisherigen Juristenausbildung aufgetretenen Mängel sowie eine von marxistischen Leerformeln gereinigte Schilderung der Zielsetzung des Reformmodells zu ersetzen. Dabei sollte dem angehenden Juristen mehr als bisher vor Augen geführt werden, daß Recht und Gerechtigkeit nur in bewußter Auseinandersetzung mit den Gegebenheiten der sozialen Umwelt und ihrer psychologischen Komponenten verwirklicht werden können. Im übrigen sollte auch aus der Diktion der Grundthesen des Reformmodells erkennbar werden, daß hier die Juristen für einen demokratischen Rechtsstaat ausgebildet werden. 
Der Gesamtvorstand des hessischen Richterbundes kann sich nicht vorstellen, daß sich die dem demokratischen Rechtsstaat verpflichteten Richter und Staatsanwälte bereitfinden werden, die angehenden Juristen im Geiste der "Grundthesen " des Wiesbadener Modells auszubilden.

Frankfurt/Main, Ende September 1973 Der Vorstand

II.

Diese standespolitische Stellungnahme ist gekennzeichnet durch die zur Schau getragene Richterattitüde, eine reformpolitische Initiative auf einen aus der Richterperspektive entscheidbaren Sachverhalt zu reduzieren. Die Strategie ist: nicht in die Diskussion um ein Reformvorhaben einlassen, sondern Selektion der "allein « entscheidungsrelevanten Gesichtspunkte. Was »entscheidungsrelevant" ist, bestimmt das Selbstverständnis der Be- oder Getroffenen. Sie lassen sich nicht auf Probleme ein und geben das als weltanschauliche Neutralität aus. Die angeblich zu kurz gekommene Bindung an das Gesetz (so der beurlaubte Richter Pulch - MdL - in seiner ersten Stellungnahme; vgl. FR v. 14. 9. 1973, FAZ v. 13. 9. 1973) wird als Argument durch ein traditionelles richterliches Selbstverständnis getragen; auf die Thesen und Konsequenzen des Wiesbadener Modells (WM) etwa im Rahmen der Erörterung der sozialwissenschaftlich-juristischen Eingangsstufe reagiert man nicht: dies mindestens würde »einlassen « zu bedeuten haben. Aber vielleicht hätte das zur Konsequenz, den Anschein weltanschaulicher Neutralität oder richterlicher Objektivität gegen konservative Parteinahme auswechseln und dann legitimieren zu müssen. Doch im politischen Tagesgeschäft ist eine solche "Distanzierungsstrategie " offenbar effektiver. Auf ihrer Basis lassen sich beispielsweise Einzelmeinungen zu Standesorganisationsstellungnahmen umfrisieren: Wer Fragen wie in der Entschließung formuliert, weiß die Antwort im voraus: sie lassen nur diese eine! So ergibt es sich, daß eine Stellungnahme, nämlich die des Richters Pulch, Vorsitzender des Deutschen Richterbundes Sekrion Hessen als Stellungnahme und Boykottdrohung anderen politischen Gewichts auf uns kommt und vielleicht dereinst als Stellungnahme des Deutschen Richterbundes, dessen stellvertretender Vorsitzender Richter Pulch ist, über uns kommen wird (Lehrstück: wie man den Uberbau zur Basis macht).

Um den Anschein weltanschaulicher Neutralität, der gebotenen, zu erwecken, dürfte derzeit kaum ein Mittel tauglicher sein als dem Gegner (man selbst bleibt ja neutral) >vulgär-marxistischen، Dogmatismus vorzuwerfen. Wäre die einzige Quelle der >dogmatischen` Verfasser der Thesen im Wiesbadener Modell über die Funktion der Juristen im konstitutionellen Staat das Kommunistische Manifest gewesen, so hätten sie nur schreiben können, daß die Bourgeoisie die Juristen ihres Heiligenscheins entkleidet und sie zu ihren bezahlten Lohnarbeitern gemacht hat - mehr steht über die Juristen nicht drin. Würde sich der Gesamtvorstand im Marxismus auskennen und nicht nur in der Zuschreibung von Vulgär-Marxismus, hätte er sogar in Marxens Kritik des Gothaer Programms einen Hinweis gefunden, der die These im WM einschränken könnte. Marx bemerkt nämlich dort zur Forderung der sozialistischen Arbeiterpartei Deutschlands nach unentgeltlicher Rechtspflege, daß die Ziviljustiz fast nur die besitzenden Klassen berührr ("Sollen sie auf Kosten des Volkssäckels ihre Prozesse führen? «). Die Justiz hat(te) also auch die Funktion, die Reibungslosigkeit des kapitalistischen Warenverkehrs zu garantieren -. ein Aspekt der 'Aufrechterhaltung von Klassenherrschafte, der nicht unmittelbar mit der Unterdrückung der Arbeiterklasse 
zusammenfällt. Aber woher dann die These, wenn nicht einfach von Marx oder V-Marx? Man muß sich schon etwas mit der Justizgeschichte beschäftigt haben, um etwa auf einen nationalliberalen Kronzeugen zu stoßen (namens Haarmann), der die Justiz pries: "Wir sind stolz auf unsere Justiz, weil sie heute noch den wirksamsten und festesten Damm bildet gegen das gemeingefährliche Treiben der Sozialdemokratie. "Man sollte sich mit Debatten im Reichstag befassen, in denen selbst Abgeordnete der Freien Volkspartei den Vorwurf der Klassenjustiz erhoben. Die zahlreichen Reden und Schriften Karl Liebknechts zum Problem der Klassen justiz sind wahrscheinlich nur für den vulgär-marxistisch, der sie nicht kennt. Die Vertreter welcher poltischen Gruppierungen stritten selbst noch I 9 I 9 in den Beratungen der Weimarer Nationalversammlung die Existenz von Klassenjustiz im Kaiserreich $a b$ ?

Klassenjustiz in der Form eines Arguments für eine höhere Richterbesoldung 1 war legitim, als Diskussionsgegenstand des Reformvorhabens einstufige Juristenausbildung in Hessen ist sie es nicht (Lehrstück: über die Verwendungsweisen von Vulgärmarxismus).

Wo sich die Vulgär-Marxisten befinden, zeigte die Debatte im Hessischen Landtag über das WM (Sitzung v. 19. 9. I 973). Der CDU-Abgeordnete Bohl sah in der Forderung nach 'Demokratisierung durch den Abbau von nicht sachbedingten Herrschaftsstrukturen< eine Übernahme der marxistischen Auffassung von der gesellschaftlichen Bedingtheit allen Rechts und damit die Anwendung der marxistischen Basis-Überbau-Theorie. Aus dem WM zitierte er: "Der Jurist ist zwar vorzüglich prädisponiert, den gesellschaftlichen " - und hier füge ich ein: vgl. die Basis-Uberbau-Theorie von Marx - »status quo zu garantieren.« Wenn von 'gesellschaftlich die Rede ist, bekommt Bohl seine Assoziationen. Die VulgärMarxisten sitzen heute in der CDU. Aber auch der Abgeordnete Pulch scheint bei seinem Marsch durch die Institutionen die FDP-Fraktion und den Vorstand des Hessischen Richterbundes mit Vulgär-Marxismus zu unterwandern.

Der hat sich auch den logischen Heuler geleistet, die Demokratie lehne die Auffassung $a b$, »daß Verhalten und Denken des Menschen durch seine Klassenlage eindeutig determiniert ist «². Diese Auffassung (bei der Herr Bohl wieder assoziiert) wird man doch wohl noch vertreten dürfen - sie wäre höchstens einzuschränken, weil von einer seindeutigen, Determination kaum die Rede sein kann -: es handelt sich jedenfalls um eine empirisch und nicht durch Berufung aufs GG entscheidbare Frage. Vielleicht ist aber gemeint, die Tatsache, daß Verhalten und Denken des Menschen durch seine Klassenlage determiniert seien, könne nicht mit demokratischen Prinzipien vereinbart werden - wer wollte dem widersprechen, wenn die Klassen undemokratisch sind. Nur, weil ein solcher Zustand undemokratisch ist, ist er nicht aus der Welt geschafft.

Warum können smarxistische Leerformeln` so viel Wirkungen zeigen? Doch wohl nicht, weil sie ein Verlangen nach Präzision und empirischem Gehalt zutiefst verletzen, sondern weil sie unseren rechten Vulgär-Marxisten, wem auch sonst, doch etwas sagen.

Dieter Hart/Hubert Rottleuthner

\footnotetext{
1 "Ohne diese (»erhebliche Weite des geistigen Horizonts«, die durch angemessene (höhere) Besoldung zu garantieren ist; d. Verf.) läuft der Richter Gefahr, sıch in seiner richterlichen Tätıgkeıt in unzulässıger Weıse mit den Wertvorstellungen und Interessen einzelner Gruppen der Gesellschaft zu identifizıeren und Entscheidungen zu treffen, die nicht im Sinne einer demokratischen und sozıalen Gesellschaftsordnung liegen, dem Grundgesetz also nicht gerecht werden." (Beschluß d. VerwG Ffm vom 25. 5. 1966, DRiZ 1966, 233, 237 in einem Verfahren, an dem Richter Pulch als Antragsteller vertreten war.)

2 Auch nach den Hess. Rahmenrichtlinien wohl: "sind"
} 